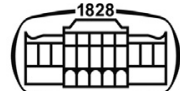

AKADÉMIAI KIADÓ

European Journal of Microbiology and Immunology

$10(2020) 3,131-138$

DOI:

$10.1556 / 1886.2020 .00011$

(c) 2020 The Author(s)

\section{ORIGINAL RESEARCH PAPER}

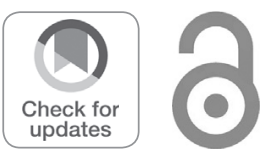

\title{
In vivo efficacy of carvacrol on Campylobacter jejuni prevalence in broiler chickens during an entire fattening period
}

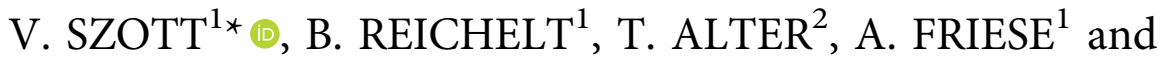 \\ U. ROESLER ${ }^{1}$ \\ ${ }^{1}$ Institute for Animal Hygiene and Environmental Health, Freie Universität Berlin, Berlin, Germany \\ ${ }^{2}$ Institute of Food Safety and Food Hygiene, Freie Universität Berlin, Berlin, Germany
}

Received: May 19, 2020 • Accepted: June 16, 2020

Published online: July 27, 2020

\section{ABSTRACT}

Carvacrol, a primary constituent of plant essential oils (EOs), and its antimicrobial activity have been the subject of many in vitro studies. Due to an increasing demand for alternative antimicrobials and an emerging number of antibiotic resistant bacteria, the use of essential oils has played a major role in many recent approaches to reduce Campylobacter colonization in poultry before slaughter age. For that purpose, the reducing effect of carvacrol on Campylobacter jejuni prevalence in broilers was determined in vivo in an experimental broiler chicken model during an entire fattening period. Carvacrol was added to the feed in a concentration of $120 \mathrm{mg} / \mathrm{kg}$ feed four days post hatch until the end of the trial. In this study, we demonstrated a statistically significant decrease of $C$. jejuni counts by 1.17 decadic logarithm $\left(\log _{10}\right)$ most probable number (MPN)/g in cloacal swabs during starter and grower periods (corresponding to a broilers age between 1 and 28 days). Similar results were observed for colon enumeration at the end of the trial where $C$. jejuni counts were significantly reduced by $1.25 \log _{10} \mathrm{MPN} / \mathrm{g}$. However, carvacrol did not successfully reduce Campylobacter cecal colonization in 33-day-old broilers.

\section{KEYWORDS}

Campylobacter jejuni, carvacrol, broiler, essential oils, feed additive, seeder bird, prevalence

\section{INTRODUCTION}

Campylobacter gastroenteritis in humans is an issue of major clinical importance worldwide [1]. Since 2005 Campylobacter is the most commonly reported zoonotic agent in the European Union (EU). In 2017, there were 246,158 reported human campylobacteriosis cases in the EU whereas Campylobacter jejuni (C. jejuni) was the most commonly isolated species and fresh broiler meat in turn the most frequent source of infection [2].

Campylobacter is highly prevalent in broiler flocks with $60-80 \%$ of the flocks being affected [3-5]. The bacterium preferably colonizes the cecal and cloacal crypts as well as the colon with high prevalence up to $10^{9}$ colony forming units (cfu)/g [6-8]. In broiler chickens, C. jejuni colonization is related to possible carcass contamination during the slaughtering process $[3,9,10]$. Even small amounts of cecal content suffice to contaminate poultry products [10]. As a result, already a $2 \log _{10}$ reduction of $C$. jejuni counts on broiler carcasses is estimated to substantially decrease the risk of human campylobacteriosis [9-13].

Although there are many approaches to reduce Campylobacter prevalence in broilers (such as feed additives, pre- and probiotics, vaccination, bacteriocins and bacteriophages), none of them has proven to be sufficient so far [10, 12, 14-17], leading to a need for further studies. Even more concerning, a recently published report stated that in the EU C. jejuni shows increasing resistance levels to fluoroquinolones $(57.7 \%)$ and tetracyclines $(45.5 \%)$ in 
humans. These levels of resistance are classified as high [18] which supports and highlights the need to develop alternative antimicrobials.

Essential oils, especially their phenolic compounds, revealed to have an antimicrobial effect against various bacteria [19, 20]. Their antimicrobial activity can be explained by their hydrophobicity $[21,22]$ and their ability to disintegrate the outer membrane of bacteria [23-25]. Moreover, studies indicated that EOs could also alter the mucosal layer through microbiome modulation [26-29]. Therefore, the use of EOs has become a promising alternative to the conventional antimicrobials $[29,30]$.

Furthermore, previous studies indicate that Campylobacter prefers a characteristic amino acid pattern for its metabolism [31, 32] since it is not dependent on carbohydrate fermentation. Due to protein-rich diets the broilers' ceca contain large amounts of these specific amino acids facilitating Campylobacter colonization [29, 33-35]. It is suggested that EOs modulate and influence ileal amino acid absorption [36] which could confine an essential source of nutrients of Campylobacter [29, 37]. As a result, it seems possible that such lack of nutrients could effectively reduce cecal Campylobacter colonization. Moreover, supplementation of EOs has been described to have beneficial effects on body weight, growth rate and feed conversion [38-41]. A supplementation is able to improve feed digestibility [42] and nutrient absorption [29].

Carvacrol is a component of many different EOs, e.g. thyme and oregano oil, and its antimicrobial activity was examined against Campylobacter in various in vitro studies $[15,22,43]$.

A previous in vitro study indicates that carvacrol is able to suppress C. jejuni in cecal content [20]. However, it has not been sufficiently established whether carvacrol can be used to reduce the $C$. jejuni prevalence in broiler chickens [13]. For this reason, an in vivo study with a seeder bird model was performed aiming to evaluate the ability of carvacrol to reduce C. jejuni colonization in broiler chickens if supplemented during an entire fattening period.

\section{MATERIALS AND METHODS}

\section{Study design}

The trials were carried out in the experimental animal facility of the Centre for Infection Medicine of the Department for Veterinary Medicine of Freie Universität Berlin. For the experiments, in total 180 broiler hatching eggs (aerosol disinfected with formaline) of breed Ross 308 were obtained from a commercial hatchery. The eggs were incubated for 21 days until hatch. Meanwhile all facilities were cleaned, disinfected (by evaporated $\mathrm{H}_{2} \mathrm{O}_{2}$ ) and tested for the absence of Campylobacter by taking various gauze swabs soaked in sterile phosphate-buffered saline (PBS; Oxoid, Wesel, Germany). Gauze swabs were processed according to DIN EN ISO 10272 and found to be free of Campylobacter.
Prior to the beginning of the trial, the 180 broiler chickens (males and females) were divided into two groups ( $n=90$ per group): a positive control group (challenged with Campylobacter and not treated - T1) and a carvacrol group (challenged with Campylobacter and treated with the experimental feed additive - T2). Immediately after hatch, 90 chickens per group were placed on ground floor with litter at a stocking density of $39 \mathrm{~kg} / \mathrm{m}^{2}$ in order to imitate a commercial broiler chicken husbandry environment. The experimental facility provided filtered air, temperature control maintained by an electronic thermometer sensor and a programmable light regimen. Broilers had access to commercial broiler feed and water ad libitum throughout the study period.

On day 1 of age, each chick was randomly assigned with an individual consecutive number for distinguishing between seeders $(n=18)$, sentinels $(n=36)$ and stocking density broilers $(n=36)$.

On day 10 of age, the seeders were orally challenged with approximately $10^{4} \mathrm{cfu} / 500 \mu \mathrm{L}$ of $C$. jejuni aiming to reproduce a natural way of infection within the broilers, as the bacteria will distribute from the seeders to the contact animals (sentinels and stocking density broilers).

In order to examine the effect of carvacrol, broilers were fed a standard diet (starter, grower, finisher) until the end of fattening (average weight $2.0 \mathrm{~kg}$ ) whereas the feed of the carvacrol group was supplemented with $120 \mathrm{mg} / \mathrm{kg}$ feed of carvacrol (Sigma-Aldrich, Munich, Germany), each.

During the trials, animal health parameters, feed intake and weight gain were monitored and recorded daily to observe possible carvacrol effects. At the end of the trial, broiler chickens were euthanized and cecal and colon contents of the sentinels were collected for Campylobacter enumeration.

\section{Bacterial strain and broiler inoculation}

The C. jejuni reference strain BfR-CA-14430 was isolated from poultry origin (chicken breast) and was provided by the Federal Institute for Risk Assessment (BfR). The strain is characterized by whole genome sequencing (WGS) and multilocus sequence typing (MLST). It belongs to the ST-21 complex. Campylobacter stock cultures were grown for $24 \mathrm{~h}$ at $37{ }^{\circ} \mathrm{C}$ under microaerophilic conditions (85\% nitrogen, $10 \%$ carbon dioxide, 5\% oxygen) in Preston Broth (PB) supplemented with Preston Campylobacter selective Supplement (SR0117; Oxoid, Wesel, Germany), Growth Supplement (SR0232; Oxoid, Wesel, Germany) and defibrinated horse blood (SR0050; Oxoid, Wesel, Germany) and then stored at $-80{ }^{\circ} \mathrm{C}$ in microbank vials (Mast Diagnostica, Germany). 48 hours before inoculation the strain was freshly recovered from frozen stocks and streaked out on Columbia Blood Agar (CBA) with 5\% sheep blood (Fisher Scientific, Germany). Plates were incubated at $37{ }^{\circ} \mathrm{C}$ under microaerophilic conditions in a tri-gas incubator (CB 160; Binder, Germany). After incubation, single colonies were resuspended in $4.0 \mathrm{~mL}$ Muller-Hinton Broth (MHB) (Oxoid, Wesel, Germany) to achieve an optical density of 0.4 at a 
wavelength of $600 \mathrm{~nm}\left(\mathrm{OD}_{600}\right)$ in order to obtain an inoculum containing $1 \times 10^{6} \mathrm{cfu} / \mathrm{mL}$. This suspension was diluted two times 1:10 in $\mathrm{MHB}$ to receive an inoculum amounting to approximately $10^{4} \mathrm{cfu} / \mathrm{mL}$. The inoculum was filled in $1 \mathrm{~mL}$ syringes and closed with plugs. Immediately after, seeders where orally challenged with $0.5 \mathrm{~mL}$ of the prepared bacteria suspension. The dose necessary for colonization was determined in previous dose-finding experiments (data not shown). For enumeration of C. jejuni, 10fold dilutions were plated on modified Campylobacter-selective charcoal cefoperazone deoxycholate agar (CCDA) plates (CM0739; Oxoid, Wesel, Germany) supplemented with CCDA selective supplement (SR0155; Oxoid, Wesel, Germany) and incubated $48 \mathrm{~h}$ at $37{ }^{\circ} \mathrm{C}$ under microaerophilic conditions.

\section{Experimental diets and admixture of carvacrol}

A three-phase feeding program diet for broilers matching the commercial standard served as experimental diet as shown in Table 1. Starter diet was offered to the broilers up to day 8 of age, followed by a grower diet until one week before necropsy (day 9-26) and a finisher diet, which was fed from day 27-33. To examine the effect of carvacrol, the carvacrol group (T2) was fed, with $120 \mathrm{mg} / \mathrm{kg}$ feed of carvacrol with a purity of $>98 \%$ beginning at day four of age. To ensure uniform mixing, the carvacrol was vaporized in a small amount of feed and then carefully mixed with the rest of the feed. In order to decrease destabilizing effects, $25.0 \mathrm{~kg}$ of the carvacrol-supplemented feed was prepared on demand and stored in airtight containers.

\section{Sampling design and sampling preparation}

On day 4 of age, all animals were monitored for Campylobacter by taking cloacal swabs (Sarstedt, Nümbrecht, Germany). For qualitative detection, the swabs were processed according to DIN EN ISO 10272. The swabs were

Table 1. Ingredients and nutrient contents of the experimental diets

\begin{tabular}{|c|c|c|c|}
\hline $\begin{array}{l}\text { Components, } \\
\text { per kg }\end{array}$ & $\begin{array}{l}\text { Starter diet } \\
(0-8 \text { days })\end{array}$ & $\begin{array}{l}\text { Grower diet } \\
\text { (9-26 days) }\end{array}$ & $\begin{array}{l}\text { Finisher diet } \\
\text { (27-33 days) }\end{array}$ \\
\hline $\begin{array}{l}\text { Crude protein } \\
(\%)\end{array}$ & 21.5 & 21.0 & 20.0 \\
\hline $\begin{array}{l}\text { Crude lipids } \\
(\%)\end{array}$ & 4.9 & 6.4 & 5.5 \\
\hline Crude fiber (\%) & 2.9 & 3.4 & 3.3 \\
\hline Crude ash (\%) & 5.3 & 5.1 & 4.9 \\
\hline $\mathrm{MJ} \mathrm{ME}$ & 12.4 & 12.4 & 12.4 \\
\hline Calcium (\%) & 0.9 & 0.8 & 0.8 \\
\hline $\begin{array}{l}\text { Phosphorous } \\
\text { (\%) }\end{array}$ & 0.6 & 0.55 & 0.5 \\
\hline Sodium (\%) & 0.14 & 0.14 & 0.14 \\
\hline $\begin{array}{l}\text { Methionine } \\
(\%)\end{array}$ & 0.55 & 0.50 & 0.50 \\
\hline Lysine (\%) & 1.25 & 1.15 & 1.05 \\
\hline
\end{tabular}

${ }^{\mathrm{a}}$ Megajoules of metabolizable energy. transferred into sterile tubes containing $3.0 \mathrm{~mL} \mathrm{~PB}$, incubated for 24 hours at $37{ }^{\circ} \mathrm{C}$ under microaerophilic conditions and then streaked out with $10 \mu \mathrm{L}$ inoculation loops (Sarstedt, Nümbrecht, Germany) on mCCDA agar plates followed by a 48 hours incubation under the same conditions. Afterward, the plates were examined for the absence of C. jejuni. Suspicious Campylobacter colonies were analyzed using a Bruker Microflex ${ }^{\mathrm{TM}}$ system for matrix-assisted laser desorption ionization time-of-flight mass spectrometry (MALDI-TOF MS).

In order to compare the results, the same 36 sentinels (untreated broilers) were sampled in both groups throughout the study by taking cloacal swabs at defined points in time: 48 hours, 72 hours and 96 hours post inoculation (equivalent to day 12,13 and 14 of age), subsequently twice a week (equivalent to day 8, 11, 15 and 18 post inoculation, respectively) until necropsy. Successful C. jejuni colonization of the seeders was verified by taking cloacal swabs 48 hours after inoculation. Cloacal swabs were prepared as described above and analyzed semiquantitatively according to DIN EN ISO 10272-3 to determine levels of Campylobacter colonization and load. For semiquantitative analysis cloacal swabs were homogenized for $3 \mathrm{~s}$ in $3.0 \mathrm{~mL}$ PB using a vortex shaker (VWR, Darmstadt, Germany) and afterward serially diluted 1:10 in PB. Dilutions were incubated $24 \mathrm{~h}$ at $37^{\circ} \mathrm{C}$ under microaerophilic conditions and then streaked out on mCCDA plates as described above.

On day 33 of age (average weight $2.0 \mathrm{~kg}$ ) all 36 sentinels per group were euthanized using ZKS poultry pliers (Corstechnology, Neerstedt, Netherlands) after confirming deep anesthesia. The animals were dissected and intestinal content (cecum and colon) was collected for C. jejuni enumeration and determination. Necropsy samples were prepared to perform semiquantitative analysis according to DIN EN ISO 10272-3. For that purpose, intestinal contents were removed sterile and diluted 1:8 in PB. After homogenization, a 10 -fold dilution series was prepared in $\mathrm{PB}$. For enrichment, diluted intestinal samples were incubated $24 \mathrm{~h}$ at $37^{\circ} \mathrm{C}$ under microaerophilic conditions, then streaked out on mCCDA plates and incubated as described above.

\section{Statistical analysis}

The experimental data was analyzed using SPSS software version 25.0 for Windows (SPSS, Inc., Chicago, IL, United States). The sample size of 36 animals was chosen to obtain statistical representative results. Campylobacter counts were logarithmically transformed $\left(\log _{10}\right)$ and then analyzed for significant differences using the non-parametric MannWhitney $U$ test. For each sampling, the mean obtained from the treated group was compared to the mean received from the control group. $P$-values below 0.05 were regarded statistically significant.

\section{Ethics}

All experimental animal procedures were approved in accordance with the German Animal Welfare Act by the State Office of Health and Social Affairs Berlin, Germany 
(Landesamt für Gesundheit und Soziales Berlin, LAGeSo) under the registration number G 0098/18. The study was conducted according to the institutional guideline for animal welfare of the Freie Universität Berlin.

\section{RESULTS}

The semiquantitative results of the control and the carvacrol group are shown in Fig. 1. Campylobacter counts are presented in $\log _{10}$ most probable number $(\mathrm{MPN}) / \mathrm{g}$. Before oral inoculation all broilers were found to be free from Campylobacter as bacteriological analysis revealed no detectable Campylobacter growth. Given that the 36 sentinels in both groups were positive for $C$. jejuni 8 days post inoculation (pi) dietary treatment with carvacrol was not able to delay C. jejuni colonization. Nevertheless, Campylobacter counts in cloacal swabs were significantly and consistently reduced $(P \leq 0.02)$ for the carvacrol group in comparison to the control group at any point in time $(8$, 11,15 and 18 days pi) (Fig. 1A). Eight and 11 days pi the highest difference in $C$. jejuni counts between the treated group (mean value 4.2 and $4.6 \log _{10} \mathrm{MPN} / \mathrm{g}$ ) and the control group (mean value 5.2 and $5.8 \log _{10} \mathrm{MPN} / \mathrm{g}$ ) could be observed. These results correspond to a mean reduction of $\geq 1 \log _{10} \mathrm{MPN} / \mathrm{g}$ (at $8 \mathrm{~d}$ pi $P<0.0001 ; r=0.53$ and at $11 \mathrm{~d}$ pi $P<0.0001 ; r=0.45)$ respectively. 15 and 18 days pi carvacrol treated animals still had significantly decreased bacterial counts (5.1 and $5.4 \log _{10} \mathrm{MPN} / \mathrm{g}$ ) in comparison to the control group (5.8 and $5.97 \log _{10} \mathrm{MPN} / \mathrm{g}$ ). However, mean reduction was $<1 \log _{10} \mathrm{MPN} / \mathrm{g}$ (at $15 \mathrm{~d}$ pi $P<0.0001$; $r=0.46$ and at $18 \mathrm{~d}$ pi $P=0.02 ; r=0.28$ ).

Nevertheless, carvacrol feed supplementation failed to reduce Campylobacter cecal colonization (Fig. 1B). Comparing both groups, $C$. jejuni counts in the ceca showed no significant difference $(P>0.05)$. However, significantly reduced $C$. jejuni numbers in the colon of carvacrol treated animals could be observed $(P<0.0001 ; r=0.53)$ in comparison to the control group (Fig. 1B).

In addition, carvacrol did not have an effect on the animals' growth performance. In comparison to the control group, the treated group showed no significant difference $(P$ $>0.05$ ) regarding weight gain and carcass weight (data not shown).

\section{DISCUSSION}

There are a few in vivo approaches which recently described the effects of carvacrol usage $[13,15,41]$. Nevertheless, to our knowledge none of these trials have been conducted in a seeder bird model and during an entire fattening period until slaughter age. In this study, we analyzed the ability of carvacrol to reduce Campylobacter carriage in broilers if supplemented to the feed in a concentration of $120 \mathrm{mg} / \mathrm{kg}$ throughout an entire fattening period. By using a seeder bird model, we aimed to achieve a better comparability with conventional animal husbandries. In particular, we aimed to evaluate the in vivo effect of carvacrol on Campylobacter prevalence on a herd and individual broiler level.

With regard to the applied dosage it is to note that carvacrol is so far only approved in one single commercial zootechnical feed additive for chicken fattening in an average amount of $5 \mathrm{mg} / \mathrm{kg}$ feed under Commission Implementing Regulation (EU) 2015/1490. However, instead of using this exact amount we decided to apply carvacrol in a 24 times higher concentration since this dosage has already shown promising reducing effects on $C$. jejuni colonization in a previous in vitro and in vivo study [41]. In vitro results yielded by using a gentamicin protection assay revealed that the presence of carvacrol $(120 \mathrm{mg} / \mathrm{kg}$ feed) significantly reduced the adhesion and invasion of a highly virulent $C$. jejuni RC039 isolate to chicken intestinal primary cells. Obviously, there is a huge discrepancy between the amount used in this study and the minimum inhibitory
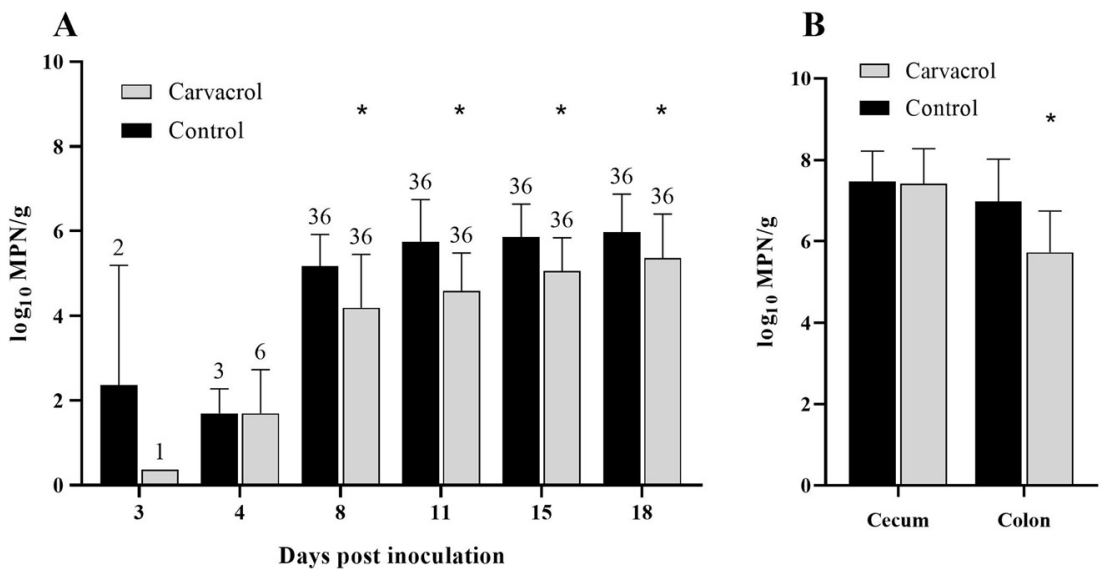

Fig. 1. (A) Mean $\log _{10}$ most probable number (MPN) of C. jejuni per gram in cloacal swabs at each point in time after inoculation. (B) Mean $\log _{10}$ MPN of $C$. jejuni per gram in intestinal content. Black bars represent the control group (broilers challenged with $C$. jejuni and not treated with carvacrol); gray bars represent broilers challenged with C. jejuni and treated with $120 \mathrm{mg} / \mathrm{kg}$ feed of carvacrol. The data presented was obtained from 36 broilers/group after necropsy. Bars marked by an asterisk differ significantly $(P<0.05)$. 
concentration (MIC) of carvacrol against C. jejuni determined in previous in vitro studies [15, 44, 45]. However, the determined MIC values for carvacrol against C. jejuni vary considerably $(0.006-0.2 \%)$ depending on the employed techniques [45] and the MIC definitions [46] used. Also, in practice, carvacrol can only be applied in relatively low concentrations, given that already dietary carvacrol in the amount of $200 \mathrm{mg} / \mathrm{kg}$ significantly lowers the broilers' feed intake and weight gain [47], highlighting that a MIC of carvacrol necessary to inhibit C. jejuni may compromise animal health as well as growth performance. Finally, Alphen et al. [48] demonstrated in an in vitro study that even sub-inhibitory concentrations (subMIC) of carvacrol $(0.2 \mathrm{mM})$ which do not affect bacterial growth are able to attenuate C. jejuni virulence and protect against cellular infection.

The results of this in vivo study demonstrate that a supplementation with $120 \mathrm{mg} / \mathrm{kg}$ feed of carvacrol is able to reduce $C$. jejuni load in cloacal swabs significantly at a 0.6 $\log _{10}$ minimum. As McLendon et al. [49] enlightened in a recent study, the use of cloacal swabs is a reliable method to detect Campylobacter. Also, Glünder [50] reported a high correlation between the analysis of cloacal swabs and cecal content. Therefore, we assume that the bacteria prevalence determined by cloacal swabs is representative for cecal $C$. jejuni colonization. Moreover, we could observe a significant C. jejuni decrease in the broilers' colon at the end of the trial at necropsy. Since we could not only observe a consistent $C$. jejuni reduction in cloacal swabs during the entire fattening period but also in the colon at the end of the trial it is reasonable to assume that a carvacrol supplemented diet likely leads to a reduced fecal contamination with C. jejuni at slaughter and to a reduction of cecal colonization.

Nevertheless, the cecal counts showed no significant difference in 33-day-old broilers compared to the control group. A possible reason for the limited efficacy of carvacrol at the end of fattening may be the chosen dosage. Our results suggest that the selected dosage was able to decrease C. jejuni colonization during animal starter and grower periods but that at the end of fattening the dosage was insufficient. A study in which Arsi et al. [13] demonstrated that Campylobacter cecal counts were significantly reduced in 10day-old broiler chicken (orally challenged with C. jejuni on day 3 with $1 \times 10^{7} \mathrm{cfu} / \mathrm{mL}$ ) if they were fed with $1 \%$ carvacrol or an EOs combination of $0.5 \%$ carvacrol and thymol supports this assumption. Another explanation for the limited effect of carvacrol at the end of the fattening period could be a feed uptake reduction consequently leading to a lower carvacrol uptake. Broiler chicken in their starter and grower periods show a higher individual feed consumption than broilers at the end of fattening [51]. Also, a feed uptake reduction could affect the stability of the EOs due to an extended residence time in the feed as EOs are volatile and thermolabile. Depending on their structure, EOs easily oxidize $[52,53]$ possibly leading to terpenoid deterioration [53]. However, Turek and Stintzing [53, 54] reported that EOs from thyme, including carvacrol tend to have a good storage stability.
It is also possible that components in the poultry diets itself limited the efficacy of carvacrol due to an altered cecal microbiome $[15,16]$. Recent studies indicate a possible correlation between crude protein and C. jejuni colonization. Moreover, it was shown that diets based on corn or wheat containing different levels of crude protein were able to modify broiler gut viscosity and histomorphology [16] and as a result could reduce $C$. jejuni colonization [11]. In a previous study where layer hens and broiler were orally inoculated with C. jejuni 1 day post hatch and fed either with broiler or layer feed Han et al. [55] also demonstrated the possible role of crude protein. The diets differed in their composition with crude protein and fat levels in broiler feed being higher than in layer feed. It was observed that layer hens fed with broiler feed were higher colonized than layer hens fed with layer feed. It is our assumption that protein rich diets overload the carvacrol-enhanced ileal amino acid absorption capacity as described above. Moreover, the interaction of high crude proportion in poultry diets and lower feed and carvacrol uptake at the end of fattening could consequently decrease the inhibitory effect on Campylobacter cecal colonization and might therefore explain the limited effect of carvacrol at the end of fattening.

Interestingly, Kelly et al. [41] observed in an in vivo study in which naturally colonized broilers were fed with three different concentrations of carvacrol $(120,200$ and $300 \mathrm{mg} / \mathrm{kg}$ of diet) that carvacrol feed supplementation delayed Campylobacter spp. colonization as the presence of Campylobacter was only detectable at day 35 of age whereas in the control group Campylobacter was detectable already at 21 days of age. Cecal content quantification showed that treated broilers did not have significantly lower Campylobacter cecal counts at 35 days of age, which correspond with our observations. However, we were not able to observe such delay in colonization. In fact, 8 days pi all sentinels were tested positive for C. jejuni. Other in vivo studies which examined the effect of different feed additives or a feed additive containing organic acids and botanicals could not observe a delay in C. jejuni colonization either $[15,17]$. This may be due to the difference between the colonization models [56]. In our approach, we orally inoculated 18 seeders artificially. A previous mathematical model suggests that one seeder will colonize 1.04 broilers per day successfully [57]. According to the model 3 days pi all broilers should be colonized successfully. Considering the digestive time to process C. jejuni [56] it is very likely to determine the bacteria in the animals 6-8 days pi. In addition, seeders were tested positive for C. jejuni 48 hours pi, which suggests that carvacrol supplementation is not able to prevent colonization in artificially colonized broilers. As a result, it is unlikely to observe a colonization delay in sentinels since seeders already started shedding the bacterium.

For further experiments it could be beneficial to add microencapsulated EOs to the feed because this procedure prevents certain components of the EOs from being absorbed or enzymatically decomposed before reaching their destination $[13,29,58]$ and may therefore improve their 
efficacy $[13,29,30]$. In an in vivo attempt, Grilli et al. [15] administered an organic acid and EO combination microencapsulated to broiler chickens. Cecal content enumeration showed significantly reduced $C$. jejuni counts.

In addition, it appears that an enhanced in vivo efficacy of EOs may be achieved through synergistic effects [15, 29, 30]. Skånseng et al. [59] performed an in vivo study in which broilers were fed a feed additive containing a combination of two acids (sorbate and formic acid) and observed similar results. While a combination of these two acids was able to prevent $C$. jejuni colonization in cecal contents completely, this effect could not be achieved by adding a single acid to the feed.

\section{CONCLUSION}

To our knowledge, this is the first in vivo study which assessed the effect of carvacrol in a seeder bird model and during an entire fattening period simultaneously. The present study demonstrates promising effects of carvacrol on C. jejuni colonization. Carvacrol feed supplementation decreased $C$. jejuni counts during broiler starter and grower periods while this effect could also be observed in colon contents but not in cecal contents at the end of the trial. Therefore, it is reasonable to assume that carvacrol in a concentration lower than $120 \mathrm{mg} / \mathrm{kg}$ may fail to reduce Campylobacter cecal colonization in vivo which is in contrast to the currently approved amount of carvacrol as a feed additive. In order to achieve reduced Campylobacter numbers in the cecum there are two promising options which could be worthwhile for further investigation: on the one hand, supplementing carvacrol in a combination with organic acids or other efficient supplements, on the other hand, supplementing increasing levels of carvacrol over the fattening period in relation to the broilers' body weight and their feed uptake. At the same time however, adverse effects on growth performance should be taken into account. In addition, the described experimental approach should be carried out with a subsequent experimental slaughter, in which the Campylobacter numbers in the cecum, in the colon and on the carcasses are examined.

In conclusion, successful establishment of pre-harvest interventions measures requires further in vivo examinations on the effect of carvacrol, especially on its mode of action and physiological pathways within the broilers gut. Once its effects have been understood entirely, intervention measures could target and control Campylobacter successfully and subsequently may lead to a decreased risk of human infections. If future in vivo experiments or field studies confirmed the efficacy of carvacrol to diminish Campylobacter colonization it would be desirable to approve carvacrol as a feed additive in higher amounts. In addition, verification of the commercial suitability and economic efficiency of the use of carvacrol as a feed additive for the poultry industry is needed.
Funding sources: The project was supported by funds of the Federal Ministry of Education and Research (BMBF) (Grant number: 01KI1725A). In addition, we acknowledge support by the German Research Foundation and the Open Access Publication Fund of the Freie Universität Berlin.

Authors' Contributions: VS performed the experiments, collected, analyzed and interpreted the data, and drafted the manuscript and figures, with critical evaluation and support of all other authors. BR helped with the experiments and laboratory work. AF and UR conceived and designed the study, as well as critically revised the manuscript. All authors approved the final version to be published.

Conflicts of interest: The authors declare that they have no conflict of interest.

\section{ACKNOWLEDGMENTS}

We acknowledge the Federal Institute for Risk Assessment for providing the C. jejuni isolate for the study. Many thanks to Michael Kühl, Susann Sellenthin and Sabrina Hansen for excellent technical support. We are also pleased to acknowledge Dr. Roswitha Merle and Dr. Racem Romdhane for statistical animal experiments group scheduling.

\section{REFERENCES}

1. Kaakoush NO, Castano-Rodriguez N, Mitchell HM, Man SM. Global epidemiology of campylobacter infection. Clin Microbiol Rev 2015;28:687-720.

2. Authority EFS. The European Union summary report on trends and sources of zoonoses, zoonotic agents and food-borne outbreaks in 2017. EFSA J 2018;16:e05500.

3. Herman L, Heyndrickx M, Grijspeerdt K, Vandekerchove D, Rollier I, De Zutter L. Routes for Campylobacter contamination of poultry meat: epidemiological study from hatchery to slaughterhouse. Epidemiol Infect 2003;131:1169-80.

4. Reich F, Atanassova V, Haunhorst E, Klein G. The effects of Campylobacter numbers in caeca on the contamination of broiler carcasses with Campylobacter. Int J Food Microbiol 2008;127: 116-20.

5. Authority EFS. Analysis of the baseline survey on the prevalence of Campylobacter in broiler batches and of Campylobacter and Salmonella on broiler carcasses, in the EU, 2008-Part B: analysis of factors associated with Campylobacter colonisation of broiler batches and. EFSA J 2010;8:1522.

6. Beery JT, Hugdahl MB, Doyle MP. Colonization of gastrointestinal tracts of chicks by Campylobacter jejuni. Appl Environ Microbiol 1988;54:2365-70.

7. Corry JE, Atabay HI. Poultry as a source of Campylobacter and related organisms. Symp Ser Soc Appl Microbiol 2001;90:96S-114S.

8. Smith CK, Abuoun M, Cawthraw SA, Humphrey TJ, Rothwell L, Kaiser P, et al. Campylobacter colonization of the chicken induces 
a proinflammatory response in mucosal tissues. FEMS Immunol Med Microbiol 2008;54:114-21.

9. Rosenquist H, Nielsen NL, Sommer HM, Nørrung B, Christensen BB. Quantitative risk assessment of human campylobacteriosis associated with thermophilic Campylobacter species in chickens. Int J Food Microbiol 2003;83:87-103.

10. Hermans D, Van Deun K, Messens W, Martel A, Van Immerseel F, Haesebrouck F, et al. Campylobacter control in poultry by current intervention measures ineffective: urgent need for intensified fundamental research. Vet Microbiol 2011;152:219-28.

11. Van Deun K, Haesebrouck F, Van Immerseel F, Ducatelle R, Pasmans F. Short-chain fatty acids and L-lactate as feed additives to control Campylobacter jejuni infections in broilers. Avian Pathol 2008;37:379-83.

12. Lin J. Novel approaches for Campylobacter control in poultry. Foodborne Pathog Dis 2009;6:755-65.

13. Arsi K, Donoghue AM, Venkitanarayanan K, Kollanoor-Johny A, Fanatico AC, Blore PJ, et al. The efficacy of the natural plant extracts, thymol and carvacrol against Campylobacter colonization in broiler chickens. J Food Safety 2014;34:321-5.

14. Ghareeb K, Awad WA, Mohnl M, Porta R, Biarnes M, Bohm J, et al. Evaluating the efficacy of an avian-specific probiotic to reduce the colonization of Campylobacter jejuni in broiler chickens. Poult Sci 2012;91:1825-32.

15. Grilli E, Vitari F, Domeneghini C, Palmonari A, Tosi G, Fantinati P, et al. Development of a feed additive to reduce caecal Campylobacter jejuni in broilers at slaughter age: from in vitro to in vivo, a proof of concept. J Appl Microbiol 2013;114:308-17.

16. Molnar A, Hess C, Pal L, Wagner L, Awad WA, Husveth F, et al. Composition of diet modifies colonization dynamics of Campylobacter jejuni in broiler chickens. J Appl Microbiol 2015;118:245-54.

17. Meunier M, Guyard-Nicodeme M, Dory D, Chemaly M. Control strategies against Campylobacter at the poultry production level: biosecurity measures, feed additives and vaccination. J Appl Microbiol 2016;120:1139-73.

18. Authority EFS. The European Union summary report on antimicrobial resistance in zoonotic and indicator bacteria from humans, animals and food in 2017. EFSA J 2019;17:e05598.

19. Sokovic M, Glamoclija J, Marin PD, Brkic D, van Griensven LJ. Antibacterial effects of the essential oils of commonly consumed medicinal herbs using an in vitro model. Molecules 2010;15: 7532-46.

20. Kollanoor Johny A, Darre MJ, Donoghue AM, Donoghue DJ, Venkitanarayanan K. Antibacterial effect of trans-cinnamaldehyde, eugenol, carvacrol, and thymol on Salmonella Enteritidis and Campylobacter jejuni in chicken cecal contents in vitro. J Appl Poult Res 2010;19:237-44.

21. Nazzaro F, Fratianni F, De Martino L, Coppola R, De Feo V. Effect of essential oils on pathogenic bacteria. Pharmaceuticals (Basel) 2013;6:1451-74.

22. O'Bryan CA, Pendleton SJ, Crandall PG, Ricke SC. Potential of plant essential oils and their components in animal agriculture - in vitro studies on antibacterial mode of action. Front Vet Sci 2015;2: 35.

23. Helander IM, Alakomi H-L, Latva-Kala K, Mattila-Sandholm T, Pol I, Smid EJ, et al. Characterization of the action of selected essential oil components on gram-negative bacteria. J Agric Food Chem 1998;46:3590-5.
24. Ultee A, Kets EP, Alberda M, Hoekstra FA, Smid EJ. Adaptation of the food-borne pathogen Bacillus cereus to carvacrol. Arch Microbiol 2000;174:233-8.

25. Ultee A, Bennik MH, Moezelaar R. The phenolic hydroxyl group of carvacrol is essential for action against the food-borne pathogen Bacillus cereus. Appl Environ Microbiol 2002;68:1561-8.

26. Hansen AK, Hansen $\mathrm{CH}$, Krych L, Nielsen DS. Impact of the gut microbiota on rodent models of human disease. World J Gastroenterol 2014;20:17727-36.

27. Kaakoush NO, Sodhi N, Chenu JW, Cox JM, Riordan SM, Mitchell HM. The interplay between Campylobacter and Helicobacter species and other gastrointestinal microbiota of commercial broiler chickens. Gut Pathog 2014;6:18.

28. Thibodeau A, Fravalo P, Yergeau E, Arsenault J, Lahaye L, Letellier A. Chicken caecal microbiome modifications induced by Campylobacter jejuni colonization and by a non-antibiotic feed additive. PLoS One 2015;10:e0131978.

29. Micciche A, Rothrock MJ, , Jr., Yang Y, Ricke SC. Essential oils as an intervention strategy to reduce campylobacter in poultry production: a review. Front Microbiol 2019;10:1058.

30. Calo JR, Crandall PG, O'Bryan CA, Ricke SC. Essential oils as antimicrobials in food systems - a review. Food Control 2015;54: $111-9$.

31. Wright JA, Grant AJ, Hurd D, Harrison M, Guccione EJ, Kelly DJ, et al. Metabolite and transcriptome analysis of Campylobacter jejuni in vitro growth reveals a stationary-phase physiological switch. Microbiology 2009;155:80-94.

32. Stahl M, Butcher J, Stintzi A. Nutrient acquisition and metabolism by Campylobacter jejuni. Front Cell Infect Microbiol 2012;2:5.

33. Jozefiak D, Rutkowski A, Martin SA. Carbohydrate fermentation in the avian ceca: a review. Anim Feed Sci Technol 2004;113:1-15.

34. Vegge CS, Brondsted L, Li YP, Bang DD, Ingmer H. Energy taxis drives Campylobacter jejuni toward the most favorable conditions for growth. Appl Environ Microbiol 2009;75:5308-14.

35. Hermans D, Pasmans F, Heyndrickx M, Van Immerseel F, Martel A, Van Deun K, et al. A tolerogenic mucosal immune response leads to persistent Campylobacter jejuni colonization in the chicken gut. Crit Rev Microbiol 2012;38:17-29.

36. Jamroz D, Orda J, Kamel C, Wiliczkiewicz A, Wertelecki T, Skorupińska J. The influence of phytogenic extracts on performance, nutrient digestibility, carcass characteristics, and gut microbial status in broiler chickens. J Anim Feed Sci 2003;12: 583-96.

37. Velayudhan J, Jones MA, Barrow PA, Kelly DJ. L-serine catabolism via an oxygen-labile L-serine dehydratase is essential for colonization of the avian gut by Campylobacter jejuni. Infect Immun 2004; 72:260-8.

38. Lee KW, Everts H, Kappert HJ, Frehner M, Losa R, Beynen AC. Effects of dietary essential oil components on growth performance, digestive enzymes and lipid metabolism in female broiler chickens. Br Poult Sci 2003;44:450-7.

39. Jang IS, Ko YH, Yang HY, Ha JS, Kim JY, Kim JY, et al. Influence of essential oil components on growth performance and the functional activity of the pancreas and small intestine in broiler chickens. Asian-Australasian J Anim Sci 2004;17:394-400.

40. Hernández F, Madrid J, García V, Orengo J, Megías MD. Influence of two plant extracts on broilers performance, digestibility, and digestive organ size 1. Poult Sci 2004;83:169-74. 
41. Kelly C, Gundogdu O, Pircalabioru G, Cean A, Scates P, Linton $\mathrm{M}$, et al. The in vitro and in vivo effect of carvacrol in preventing campylobacter infection, colonization and in improving productivity of chicken broilers. Foodborne Pathog Dis 2017;14: 341-9.

42. Williams P, and Losa, R. The use of essential oils and their compounds in poultry nutrition. Worlds Poult Sci J 2001;17:14-5.

43. Friedman M, Henika PR, Mandrell RE. Bactericidal activities of plant essential oils and some of their isolated constituents against Campylobacter jejuni, Escherichia coli, Listeria monocytogenes, and Salmonella enterica. J Food Prot 2002;65:1545-60.

44. Ravishankar S, Zhu L, Law B, Joens L, Friedman M. Plant-derived compounds inactivate antibiotic-resistant Campylobacter jejuni strains. J Food Prot 2008;71:1145-9.

45. Navarro M, Stanley R, Cusack A, Sultanbawa Y. Combinations of plant-derived compounds against Campylobacter in vitro. J Appl Poult Res 2015;24:352-63.

46. Burt S. Essential oils: their antibacterial properties and potential applications in foods - a review. Int J Food Microbiol 2004;94: 223-53.

47. Lee KW, Everts H, Kapperst HJ, Yeom KH, Beynen AC. Dietary carvacrol lowers body weight gain but improves feed conversion in female broiler chickens. J Appl Poult Res 2003;12:394-9.

48. van Alphen LB, Burt SA, Veenendaal AK, Bleumink-Pluym NM, van Putten JP. The natural antimicrobial carvacrol inhibits Campylobacter jejuni motility and infection of epithelial cells. PLoS One 2012;7:e45343.

49. McLendon BL, Cox NA, Cosby DE, Montiel ER, Russell SM, Hofacre CL, et al. Detecting Campylobacter coli in young chicks using two different cloacal swab techniques. J Appl Poult Res 2018; 27:223-7.
50. Glunder G. Infectivity of Campylobacter jejuni and Campylobacter coli in chickens. Berl Munch Tierarztl Wochenschr 1995;108:101-4.

51. Siegmann O, Neumann U, Kamphues J. Kompendium der Geflügelkrankheiten. Schlütersche; 2012.

52. Scott RPW. Essential oils. In: Worsfold P, Townshend A, Poole C, editors. Encyclopedia of analytical science (2nd ed.). Oxford: Elsevier; 2005:554-61 pp.

53. Turek C, Stintzing FC. Stability of essential oils: a review. Compr Rev Food Sci Food Safety 2013;12(1):40-53.

54. Turek C, Stintzing FC. Evaluation of selected quality parameters to monitor essential oil alteration during storage. J Food Sci 2011;76: C1365-75.

55. Han Z, Willer T, Pielsticker C, Gerzova L, Rychlik I, Rautenschlein S. Differences in host breed and diet influence colonization by Campylobacter jejuni and induction of local immune responses in chicken. Gut Pathog 2016;8:56.

56. Conlan AJ, Coward C, Grant AJ, Maskell DJ, Gog JR. Campylobacter jejuni colonization and transmission in broiler chickens: a modelling perspective. J R Soc Interface 2007;4:819-29.

57. Van Gerwe TJ, Bouma A, Jacobs-Reitsma WF, van den Broek J, Klinkenberg D, Stegeman JA, et al. Quantifying transmission of Campylobacter spp. among broilers. Appl Environ Microbiol 2005; 71:5765-70.

58. Van Immerseel F, Fievez V, de Buck J, Pasmans F, Martel A, Haesebrouck F, et al. Microencapsulated short-chain fatty acids in feed modify colonization and invasion early after infection with Salmonella enteritidis in young chickens. Poult Sci 2004;83:69-74.

59. Skanseng B, Kaldhusdal M, Moen B, Gjevre AG, Johannessen GS, Sekelja $M$, et al. Prevention of intestinal Campylobacter jejuni colonization in broilers by combinations of in-feed organic acids. J Appl Microbiol 2010;109:1265-73. 